\title{
Biomarker-Based Phase II Trial of Savolitinib in Patients With Advanced Papillary Renal Cell Cancer
}

Toni K. Choueiri, Elizabeth Plimack, Hendrik-Tobias Arkenau, Eric Jonasch, Daniel Y.C. Heng, Thomas Powles, Melanie M. Frigault, Edwin A. Clark, Amir A. Handzel, Humphrey Gardner, Shethah Morgan, Laurence Albiges, and Sumanta Kumar Pal

Author affiliations and support information (if applicable) appear at the end of this article.

Published at jco.org on June 23, 2017

Processed as a Rapid Communication manuscript.

Clinical trial information: NCT02127710.

Corresponding author: Toni K. Choueiri, MD, Lank Center for Genitourinary Oncology, Department of Medical Oncology, Dana-Farber Cancer Institute, 450 Brookline Ave, Dana 1230, Boston, MA 02215; e-mail: toni_choueiri@dfci. harvard.edu.

(C) 2017 by American Society of Clinical Oncology

0732-183X/17/3599-1/\$20.00
ASSOCIATED CONTENT

(2) Appendix

DOI: https://doi.org/10.1200/JCO 2017.72.2967

DOI: https://doi.org/10.1200/JCO.2017. 72.2967

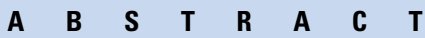

\section{Purpose}

Patients with advanced papillary renal cell carcinoma (PRCC) have limited therapeutic options. PRCC may involve activation of the MET pathway, for example, through gene amplification or mutations. Savolitinib (AZD6094, HMPL-504, volitinib) is a highly selective MET tyrosine kinase inhibitor. We report results of a single-arm, multicenter, phase II study evaluating the safety and efficacy of savolitinib in patients with PRCC according to MET status.

\section{Patients and Methods}

Patients with histologically confirmed locally advanced or metastatic PRCC were enrolled and received savolitinib $600 \mathrm{mg}$ orally once daily. MET-driven PRCC was defined as any of the following: chromosome 7 copy gain, focal MET or HGF gene amplification, or MET kinase domain mutations. Efficacy was assessed according to MET status. Safety, toxicity, and patient-reported health-related quality-of-life outcomes were assessed in all patients.

\section{Results}

Of 109 patients treated, PRCC was MET driven in 44 (40\%) and MET independent in 46 (42\%); MET status was unknown in 19 (17\%). MET-driven PRCC was strongly associated with response; there were eight confirmed partial responders with MET-driven disease (18\%), but none with METindependent disease $(P=.002)$. Median progression-free survival for patients with MET-driven and MET-independent PRCC was 6.2 months (95\% Cl, 4.1 to 7.0 months) and 1.4 months (95\% Cl, 1.4 to 2.7 months), respectively (hazard ratio, $0.33 ; 95 \% \mathrm{Cl}, 0.20$ to 0.52 ; log-rank $P<.001$ ). The most frequent adverse events associated with savolitinib were nausea, fatigue, vomiting, and peripheral edema.

\section{Conclusion}

These data show activity and tolerability of savolitinib in the subgroup of patients with MET-driven PRCC. Furthermore, molecular characterization of MET status was more predictive of response to savolitinib than a classification based on pathology. These findings justify investigating savolitinib in MET-driven PRCC.

\section{J Clin Oncol 35. () 2017 by American Society of Clinical Oncology}

\section{INTRODUCTION}

Renal cell carcinoma (RCC) is a heterogeneous disease comprising several histologic subtypes with different genetic and biochemical characteristics; clear cell RCC is the most frequent, accounting for $75 \%$ to $90 \%$ of renal malignancies. ${ }^{1}$ Of the non-clear cell renal carcinomas, papillary RCC (PRCC) is the most common, with a recent study of patients with metastatic nonclear cell RCC reporting $40 \%$ as papillary by histology, followed by chromophobe RCC ( $\leq 5 \%)$ and other less common subtypes. ${ }^{1-3}$ In 2017 , it is estimated 64,000 new cases of RCC will be diagnosed in the United States, equating to up to 6,400 cases of PRCC. ${ }^{4}$

Somatic PRCC is conventionally classified into two histologic subtypes (type 1 and type 2), with a worse prognosis reported for type $2 .^{5-9}$ Molecular profiling of PRCC corroborates disease linkage demonstrated by studying rare hereditary syndromes that lead to RCC. MET mutations are associated with hereditary papillary renal carcinoma and phenocopy PRCC type 1 histologies, whereas fumarate hydratase mutations in hereditary leiomyomatosis RCC are associated with a subset of papillary type 2 histologies. ${ }^{10}$ 
Molecular classification based on MET or hepatocyte growth factor (HGF) aberrations may provide more clinically relevant and reproducible indicators of tumor behavior than histologic subtype. Two recent cohorts of molecular analyses of PRCC have confirmed the utility of molecular features to reclassify this disease. ${ }^{11,12}$

Activation of the HGF/MET axis triggers tumor growth, promotes angiogenesis, and induces metastasis. ${ }^{13}$ MET activation is also associated with treatment resistance ${ }^{14-16}$ and correlates with poor prognosis in many tumor types, including clear cell RCC. ${ }^{17-21}$ Germline mutations in the MET gene, found on chromosome $7 q 31$, were first described in patients with hereditary PRCC. In addition to MET mutations, ${ }^{22-24}$ copy number gain of chromosome 7 (containing loci of both the MET receptor gene, MET, and its ligand, $H G F$ ) is common, occurring in $45 \%$ to $75 \%$ of sporadic PRCC cases, and copy number alterations of MET occur in $81 \%$ of type 1 and $46 \%$ of type 2 PRCCs. ${ }^{11,12}$

Currently, prognosis for patients with advanced PRCC is poor, because of the limited efficacy of currently available therapies, which were mainly developed for clear cell RCC. In studies of patients with PRCC, a majority of whom were previously untreated, treatment with the multireceptor tyrosine kinase inhibitor sunitinib typically showed an objective response rate (ORR) of less than $10 \%$ and a median progression-free survival (PFS) of approximately $\leq 6$ months. ${ }^{25-29}$ These data compare unfavorably with an ORR of $25 \%$ to $30 \%$ and a median PFS of 9.5 to 11 months for patients with treatment-naive clear cell RCC receiving sunitinib. $^{30-33}$ The activity of foretinib, a multikinase inhibitor targeting MET, vascular endothelial growth factor, RON, AXL, and TIE-2 receptors, against PRCC was assessed in a phase II study. ${ }^{34}$ Tumor responses were reported in five of 10 patients with germline MET mutations, compared with five of 57 patients without such a mutation, suggesting potential benefit for patients with METdriven PRCC.

Savolitinib (AZD6094, HMPL-504, volitinib) is a potent, selective MET inhibitor being investigated as a targeted therapy for patients with non-small-cell lung cancer in combination with osimertinib, ${ }^{35}$ as well as for patients with advanced or metastatic PRCC. On the basis of preclinical data and a phase I study showing partial responses in three of six patients with PRCC, ${ }^{36,37}$ a singlearm, multicenter, phase II study was conducted to evaluate the safety and efficacy of savolitinib in patients with PRCC and to correlate savolitinib activity with MET pathway alterations.

\section{PATIENTS AND METHODS}

\section{Patients}

Eligible patients had histologically confirmed locally advanced or metastatic PRCC, with central and/or local laboratory-confirmed pathology on pretreatment archival tumor tissue. Papillary histology was independently reviewed by two pathologists; if a consensus was not reached, a third review was performed by a pathologist at an independent institute. Other inclusion criteria included: Eastern Cooperative Oncology Group performance status of 0 or 1 , life expectancy $\geq 12$ weeks, age $\geq 18$ years, and adequate hematologic, hepatic, and renal function. Exclusion criteria included prior or current MET inhibitor treatment, at the discretion of the study monitor. One patient who had received foretinib 3.5 years earlier was included. The first patient was dosed on May 21, 2014.
All patients provided written informed consent. The study was performed in accordance with ethical principles originating in the Declaration of Helsinki and was consistent with International Conference on Harmonisation/Good Clinical Practice guidelines, applicable regulatory requirements, and the AstraZeneca policy on bioethics and human biologic samples.

\section{Study Design and Objectives}

This single-arm, multicenter, global, phase II study evaluated the safety and efficacy of savolitinib in patients with PRCC irrespective of prior treatment. The primary objective was to assess savolitinib antitumor activity in patients with PRCC and by MET status, as measured by investigator assessment of ORR according to Response Evaluation Criteria in Solid Tumors (RECIST; version 1.1). ${ }^{38}$ Secondary objectives included change in target lesion tumor size from baseline and PFS (time from first dosing until objective disease progression or death resulting from any cause) in all patients and by MET status according to RECIST (version 1.1).

\section{Study Drug Administration}

Patients received savolitinib $600 \mathrm{mg}$ orally once daily, until RECIST (version 1.1) -defined progression or treatment discontinuation criteria were met. A treatment cycle was defined as 21 days.

\section{Study Assessments}

Screening and baseline assessments were obtained $\leq 28$ days before the first savolitinib dose. After baseline evaluation, objective tumor assessments were performed every 6 weeks ( \pm 7 days) for the first 12 months and every 12 weeks thereafter until disease progression. Response to treatment was assessed by ORR, stable disease, and PFS. Blood samples for pharmacokinetic, pharmocodynamic, germline DNA, and pharmacogenetic analyses were collected.

Patient-reported health-related quality-of-life outcomes and diseaserelated symptoms were collected at the start of the first three treatment cycles and then every 6 weeks, up to and including at discontinuation. The Functional Assessment of Cancer Therapy (FACT) -General, FACT Kidney Symptom Index-19 (FKSI-19), and European Quality of Life 5-Dimensions 5-Levels (EQ-5D-5L) were used.

The safety and tolerability of savolitinib were assessed according to Common Terminology Criteria for Adverse Events (version 4.03). Adverse events (AEs) and medical/surgical history were classified according to the Medical Dictionary for Regulatory Activities (version 18.1) and recorded from on or after the first dose until 30 days after the last dose of savolitinib. End of study for ORR was January 29, 2016; data cutoff for PFS was June 27, 2016.

\section{Assessment of MET Pathway Status}

Next-generation sequencing of archival tumor tissue was analyzed using a targeted 400-gene panel (version T7; Foundation Medicine, Cambridge, MA) as previously described. ${ }^{39}$ PRCC was confirmed as MET driven by identification of $M E T$ copy number gain (either chromosome 7 gain or a focal MET amplification of $\geq$ six copies), HGF gene amplification ( $\geq$ six copies), or MET kinase domain mutations (allele frequency $>5 \%$ ). The Appendix (online only) provides more information on assessment of MET status. Focal MET amplifications were confirmed by fluorescent in situ hybridization.

\section{Statistical Considerations}

The trial size was designed to detect a response rate (ORR) of greater than $10 \%$ in patients with MET-driven disease while accounting for the estimated prevalence of this target population. A sample size of 50 patients with MET-driven PRCC allowed this signal detection at a 90\% two-sided confidence level with at least $80 \%$ power assuming the true response rate was $25 \%$ or better. Analyses of outcome measures were descriptive, and 
tests for significant differences were conducted between patients with MET-driven and MET-independent PRCC.

\section{RESULTS}

\section{Patients and Treatment}

In total, 111 patients were enrolled, and 109 received at least one dose of savolitinib (treatment population). Baseline demographic and clinical characteristics are listed in Table 1. Patient demographics were as expected for a population with advanced PRCC. Most patients $(\mathrm{n}=107 ; 98 \%)$ had metastatic disease, and 49 (45\%), 80 (73\%), and $22(20 \%)$ had previously undergone systemic therapy, nephrectomy, or radiotherapy, respectively. Overall, 28 (26\%) had received sunitinib.

PRCC was MET driven in 44 patients (40\%) and MET independent in $46(42 \%)$. MET status was unknown in 19 patients $(17 \%)$. Baseline characteristics of patients with MET-driven and -independent disease were generally similar (Table 1). In 25 patients $(23 \%)$, it was not possible to define the tumor as type 1 or 2 , partly as a result of limited tissue available for central review, because typically only a single block from each patient case is provided. The proportions classified as type 1 and type 2 PRCC by central review differed according to MET status; among patients with MET-driven PRCC, 12 (27\%) were classified as type 1 and 23 (52\%) as type 2 , whereas among patients with MET-independent

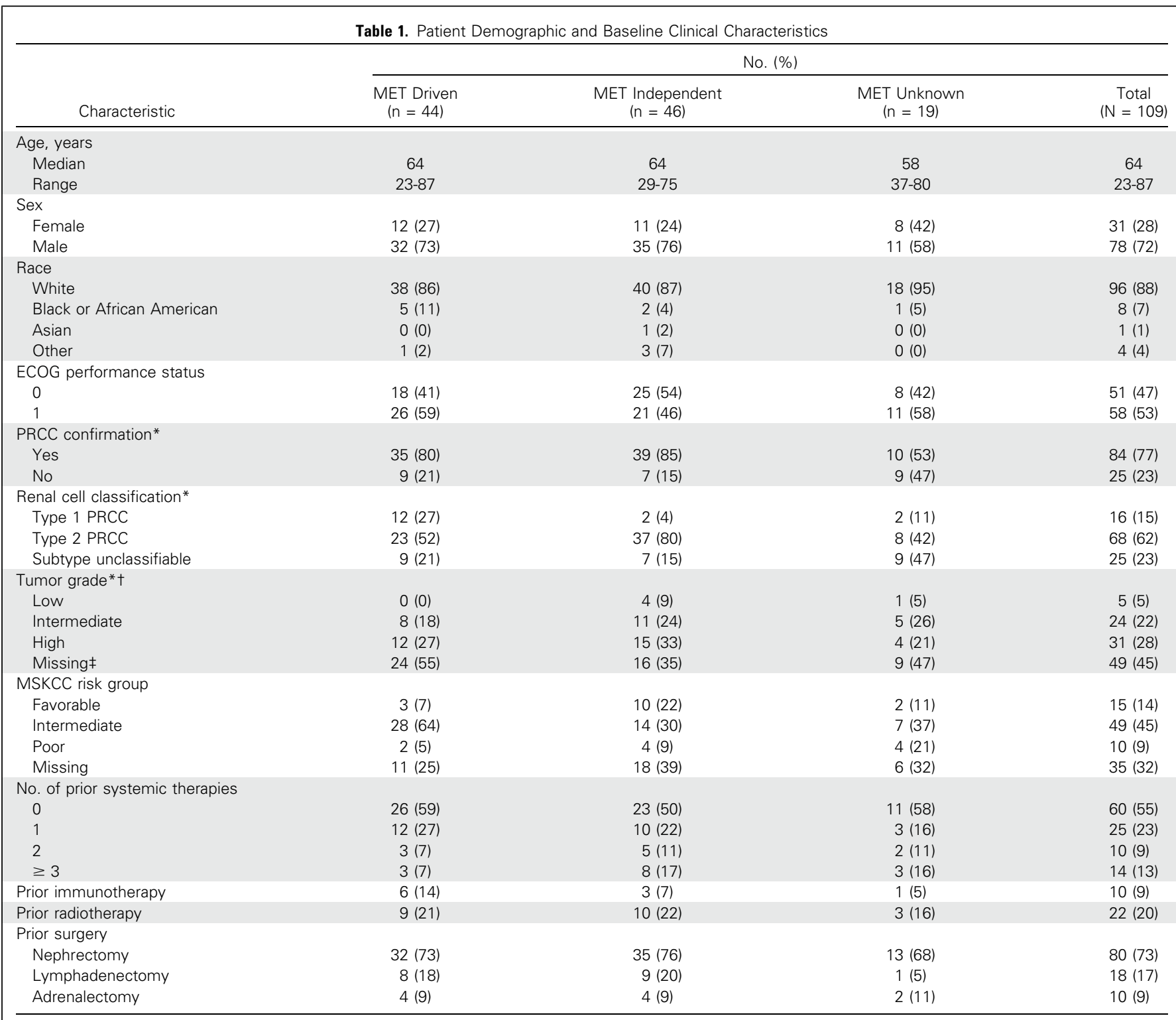

Abbreviations: ECOG, Eastern Cooperative Oncology Group; MSKCC, Memorial Sloan Kettering Cancer Center; PRCC, papillary renal cell carcinoma.

* On the basis of central laboratory data; in 25 patients, confirmation was not possible partly because of limited tissue available for central review (typically only a single block from each patient case is provided) or the subtype of PRCC was not considered classifiable.

†Modified Fuhrman nuclear grade: low, grade 1 or 2; intermediate, grade 3; and high, grade 4

¥Mainly because of missing baseline lactate dehydrogenase values. Percentages may not total $100 \%$ because of rounding. 
PRCC, the proportions were two (4\%) and 37 patients $(80 \%)$, respectively (Table 1 ).

\section{Objective Disease Response}

In the overall treatment population, the ORR was $7 \%$. However, when assessed by MET status, the ORR was significantly higher in patients with MET-driven PRCC (eight partial responses [18\%; PRs] among 44 patients) than with MET-independent PRCC (zero [0\%] PRs among 46 patients; $P=.002$; Table 2). Categorization of MET-driven PRCC by histologic subtype showed two $(17 \%)$ of 12 patients with type 1 and one $(4 \%)$ of 23 patients with type 2 PRCC exhibited a PR. Five patients with a PR were not classified as having either type 1 or 2 disease by central review.

Stable disease was achieved in 22 patients (50\%) with METdriven PRCC and 11 (24\%) with MET-independent disease (Table 2). Waterfall plots for maximal tumor response by MET status are shown in Figure 1. Of 44 patients with MET-driven PRCC, 27 (61\%) experienced some tumor shrinkage (range, $-0.7 \%$ to $-66 \%$ shrinkage), whereas nine $(20 \%)$ of 46 patients with MET-independent PRCC had any tumor shrinkage (range, $-0.5 \%$ to $-20 \%$ shrinkage).

\section{PFS and Duration of Response}

In the treatment population, 82 patients (75\%) experienced progression, died, or discontinued therapy, and 27 (25\%) continued to receive study drug or remained in follow-up at end of study. As of the June 27, 2016, data cutoff, 41 patients had died and 19 were still receiving savolitinib. Disease progression occurred in 33 (75\%), 44 (96\%), and 14 patients (74\%) with MET-driven, MET-independent, and MET-unknown PRCC, respectively. Patients with MET-driven PRCC had a significantly longer median PFS than those with MET-independent disease (6.2 months; 95\% CI, 4.1 to 7.0 months $v 1.4$ months; $95 \%$ CI, 1.4 to 2.7 months, respectively). The PFS hazard ratio (HR) for patients with METdriven PRCC was 0.33 (95\% CI, 0.20 to 0.52) compared with METindependent PRCC (log-rank $P<.001$; Fig 2$)$. After discontinuation of savolitinib, $10(23 \%)$ and 14 patients (30\%) with MET-driven and MET-independent disease, respectively, received additional lines of therapy for PRCC. Of the eight patients exhibiting a PR, six were still responding to treatment at data cutoff, with a duration of response of 2.4 to 16.4 months. Two patients who achieved

\begin{tabular}{|c|c|c|c|c|}
\hline \multirow[b]{2}{*}{$\begin{array}{c}\text { RECIST } \\
\text { Response }\end{array}$} & \multicolumn{4}{|c|}{ No. (\%) } \\
\hline & $\begin{array}{c}\text { MET } \\
\text { Driven } \\
(n=44)\end{array}$ & $\begin{array}{c}\text { MET } \\
\text { Independent } \\
(n=46)\end{array}$ & $\begin{array}{c}\text { MET } \\
\text { Unknown } \\
(\mathrm{n}=19)\end{array}$ & $\begin{array}{c}\text { Total } \\
(\mathrm{N}=109)\end{array}$ \\
\hline$P R^{*}$ & 8 (18)† & $0(0)$ & $0(0)$ & $8(7)$ \\
\hline SD & $22(50)$ & $11(24)$ & $5(26)$ & 38 (35) \\
\hline PD & $11(25)$ & $28(61)$ & $9(47)$ & $48(44)$ \\
\hline NE & $3(7)$ & 7 (15) & $5(26)$ & 15 (14) \\
\hline
\end{tabular}

Abbreviations: PR, partial response; SD, stable disease; PD, progressive disease; NE, not evaluable.

* Unconfirmed responses excluded.

$\dagger P=.002$ versus MET-independent subgroup (Fisher's exact test). Responses assessed according to RECIST (version 1.1). a PR subsequently experienced progressive disease after 1.8 and 2.8 months.

\section{Activity by Lines of Prior Therapy}

Of the patients achieving a PR, three had received no prior therapy and five had received one or more previous lines of treatment. For the 38 patients $(35 \%)$ with a best response of stable disease, 24 had received no previous therapy and 14 had received one or more prior lines of treatment. Among patients with progressive disease, 26 had received no prior therapy and 22 had received one or more lines of treatment. Prior cancer therapies are listed in Appendix Table A1 (online only).

\section{Patient-Reported Outcomes: Quality of Life}

Quality-of-life data were collected via the FACT-G, FKSI-19, and EQ-5D-5L questionnaires. Overall, during savolitinib treatment, scores for symptoms and quality of life were maintained at similar values to those reported at baseline, irrespective of the questionnaire used (Appendix Table A2, online only). This suggests that, among patients completing the questionnaires, there was little change in these outcomes during the study.

\section{Safety and Tolerability}

The overall incidence of AEs and those considered treatment related occurring in $\geq 3 \%$ of patients who received at least one dose of savolitinib are listed in Table 3. Most patients (88\%) experienced at least one $\mathrm{AE}$ considered by the investigator to be related to study drug. Abnormal liver function tests were reported in $20 \%$ of patients, irrespective of MET status. The most frequently observed hepatic AEs were increased blood AST in 12 patients (11\%) and ALT in 11 patients (10\%), which were grade 3 or higher in four $(4 \%)$ and five patients $(5 \%)$, respectively. Three patients reported serious AEs considered at least related to treatment; these were: grade 3 pneumonitis, grade 4 elevated transaminases, and grade 4 drug-induced liver injury, which led to death resulting from hepatic encephalopathy.

A total of 13 AEs in nine patients (6\%) led to drug discontinuation. These AEs included increased ALT and peripheral edema (both in two patients) and individual events of increased AST, proteinuria, pain, nausea, vomiting, fatigue, and embolism. Dosing was delayed for 47 patients (43\%); in 38 (35\%) of these patients, the delay was because of an AE. Fourteen patients (13\%) had dose reductions as a result of an $\mathrm{AE}$ at some time during the study.

\section{DISCUSSION}

To our knowledge, this is the largest study of patients with advanced or metastatic PRCC with central pathology review and biomarker analysis used to define MET-driven and METindependent PRCC. Patients experiencing responses in this study were found only in the group with MET-driven disease, indicating that savolitinib may suppress MET-driven tumor growth. Furthermore, PFS for those with MET-driven tumors was significantly longer (6.2 months) compared with those with METindependent disease (1.4 months). Continued long-term follow-up 


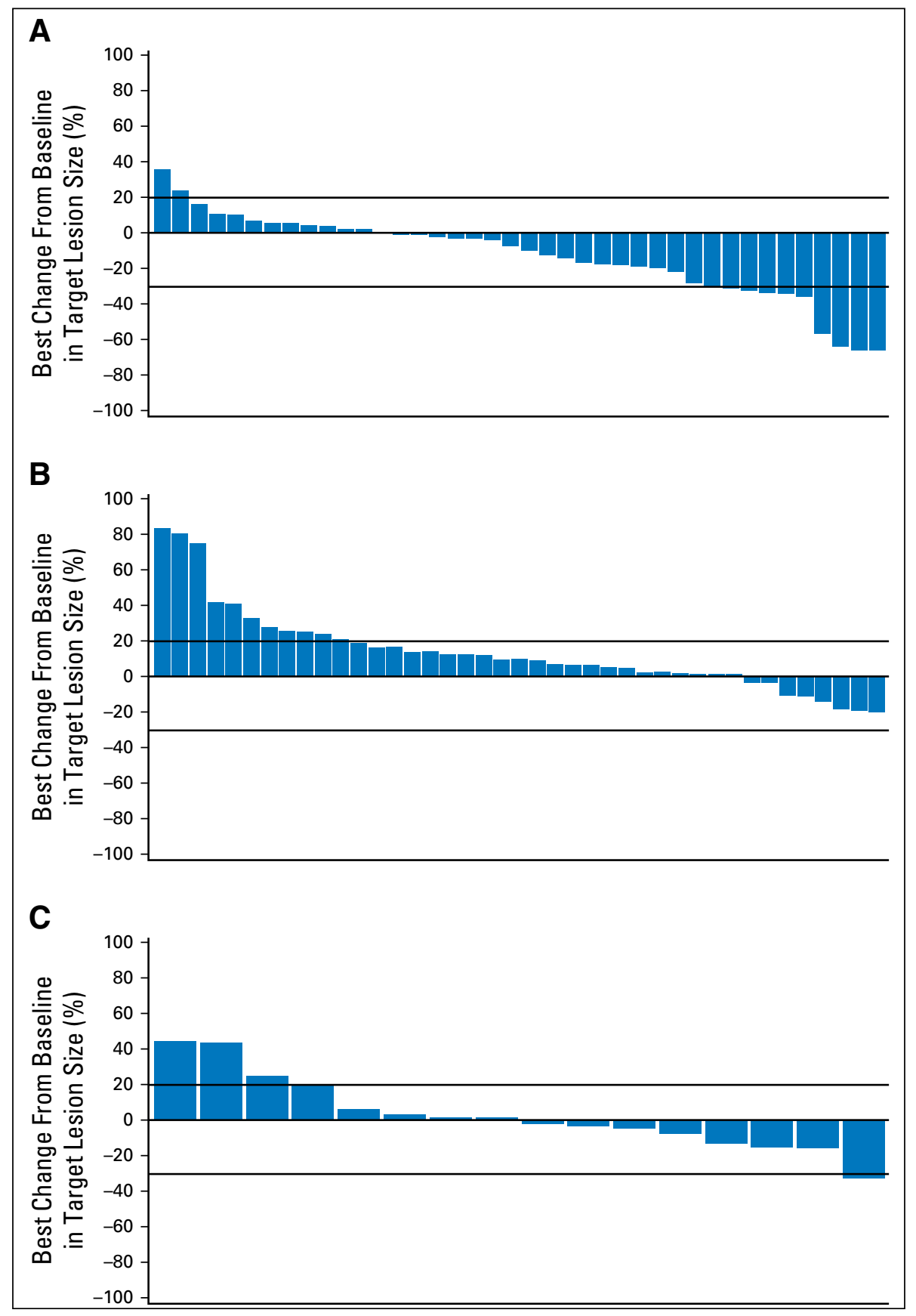

Fig 1. Best percentage change in tumor size from baseline according to MET status. Investigatorassessed measurements of target lesion size using RECIST (version 1.0) in patients with papillary renal cell carcinoma (PRCC) and MET status assessment with measurable disease at baseline and at least one postbaseline measurement. (A) MET-driven PRCC $(n=40)$; (B) MET-independent PRCC ( $n=41)$; and (C) MET status unknown $(n=16)$.

will allow further assessment of the difference in PFS and provide data on overall survival.

The analysis of MET mutations, copy number gain, or gain of chromosome 7 in patients defined as having type 1 or type 2 PRCC on the basis of pathology challenges the view that aberrations in MET are mainly associated with type 1 PRCC. Our study found MET copy number gain (either chromosomal or focal) in $72 \%$ of type 1 PRCCs and $46 \%$ of type 2 PRCCs, which is comparable to previous reports of MET copy number gain in $81 \%$ and $46 \%$ of type 1 and type 2 PRCCs, respectively. ${ }^{11}$ The frequency of MET kinase domain mutations identified here is also similar to data from The Cancer Genome Atlas Research Network of 15\%, 2\%, and $12 \%$ in type 1 , type 2 , and unclassified PRCCs, respectively. ${ }^{12}$ Of note, the The Cancer Genome Atlas publication reported any MET gene mutation, whereas our study considered only kinase domain mutations. This may explain the slightly reduced frequency of MET mutations in our study and suggests the molecular features of PRCC reported here are comparable to those of previously published cohorts. Overall, MET status was more predictive of response to savolitinib in our study than a classification based on pathology; all partial responders had archival tumor samples that harbored a copy number gain in the MET pathway (HGF, MET, or chromosome 7), some in combination with a MET kinase domain mutation. This suggests that genomic profiling can better identify 


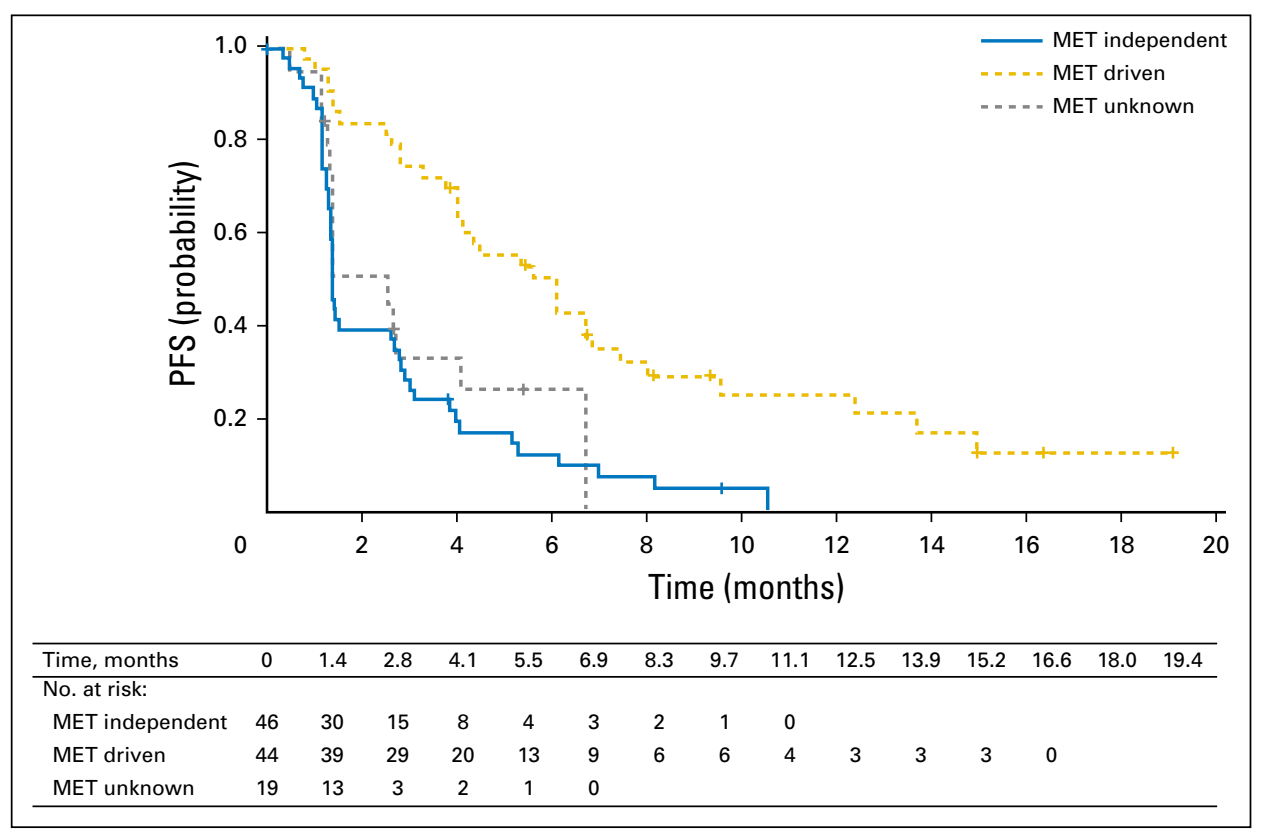

Fig 2. Kaplan-Meier estimate of progressionfree survival (PFS), defined as time from the date of first dosing until the date of objective disease progression or death resulting from any cause, in patients with papillary renal cell carcinoma by MET status (treatment population). $(+)$ indicates censored event.

patients who may respond to savolitinib than can the type 1 or type 2 histologic subtype, adding relevance to the use of genomic profiling in such studies. MET pathway PRCC tumors have also been identified by others to not only be associated with type 1 histology. $^{12}$

Overall, savolitinib was generally well tolerated, with the three most common AEs (nausea, fatigue, and vomiting) also commonly reported previously by patients with PRCC receiving foretinib. ${ }^{34}$ Other AEs occurring in $\geq 10 \%$ of patients in our study were peripheral edema, ALT and AST increases, serum creatinine increase, and decreased appetite. Increased ALT and AST have been reported in patients with PRCC treated with foretinib (in $22 \%$ and $24 \%$ of patients, respectively) and sunitinib (up to $74 \%$ of patients). ${ }^{26,27,34}$ A majority of these events, as in our study, were grade 1 or 2. Dosing of savolitinib was only reduced in four patients because of abnormal liver function, and two patients discontinued treatment because of abnormal AST or ALT levels.

Following the history of evaluating MET inhibitors for the treatment of RCC, these results with savolitinib are encouraging. There have been numerous reasons why earlier MET inhibitors have failed during drug development. For example, tivantinib was reported to be a selective MET inhibitor, but a subsequent study reported similar suppression of both MET-dependent and METindependent tumor cell lines, via inhibition of microtubule polymerization. ${ }^{40}$ Less selective multikinase inhibitors may not achieve the extent or duration of MET pathway attenuation necessary for suppression of MET-mediated migration and invasion at tolerated doses. Most importantly, patients have not been appropriately selected in the past; it is now increasingly recognized that high MET protein levels (usually detected by immunohistochemistry) do not always correlate with response or survival outcomes. ${ }^{41}$ In a phase II study of foretinib, tumor responses were reported in five of 10 patients with germline MET mutations compared with $9 \%$ of patients without MET mutations. ${ }^{34}$ Recently, in a small study of type 1 PRCC, patients with MET-driven disease (MET mutation positive [ $\mathrm{n}=4]$ and MET amplification $[\mathrm{n}=2]$ ) responded to treatment with crizotinib, an inhibitor of MET, ALK, and ROS1. ${ }^{42}$ The current interest in MET inhibitors for the treatment of PRCC is demonstrated by the initiation of a randomized phase II trial comparing the efficacy of several MET kinase inhibitors, including savolitinib (ClinicalTrials.gov identifier: NCT02761057). In that cooperative group study, patients are not being selected based on MET mutation status, although tumor response by $M E T$ mutation and expression level is an additional outcome.

Limitations of our study include the single-arm design, and therefore the inherent lack of a comparator group, and the relatively small number of patients with MET-driven disease. Prognostic information for MET in PRCC is also lacking because of the single-arm design.

In summary, these results confirm that savolitinib, a potent and selective small-molecule MET kinase inhibitor, holds promise as a personalized treatment for patients with metastatic MET-driven PRCC. Our study identified a defined molecular group and highlights the prevalence of MET-driven disease, including patients with ligand-dependent (ie, HGF amplification) and -independent PRCC who responded to treatment. These data support the hypothesis that savolitinib has antitumor activity in patients with MET-driven PRCC and justifies the recently launched phase III trial comparing savolitinib with sunitinib in a population of patients with MET-driven PRCC (ClinicalTrials. gov identifier: NCT03091192)

\section{AUTHORS' DISCLOSURES OF POTENTIAL CONFLICTS} OF INTEREST

Disclosures provided by the authors are available with this article at jco.org. 
Table 3. Overall Incidence of AEs and Those Considered Related to Savolitinib Treatment Occurring in $\geq 3 \%$ of Patients

\begin{tabular}{|c|c|c|c|}
\hline \multirow[b]{3}{*}{$A E^{*}$} & \multicolumn{3}{|c|}{ No. (\%) } \\
\hline & \multicolumn{3}{|c|}{$\begin{array}{l}\text { Treatment Population } \\
\qquad(\mathrm{N}=109)\end{array}$} \\
\hline & Grade 1-2 & Grade $\geq 3$ & Total \\
\hline Any $A E$ & $57(52)$ & $51(47)$ & $108(99)$ \\
\hline Any treatment-related $\mathrm{AE} \dagger$ & $75(69)$ & $21(19)$ & $96(88)$ \\
\hline Any SAE (including death) & & & $23(21)$ \\
\hline \multicolumn{4}{|l|}{ Death } \\
\hline Related to PRCC or disease progression & & & $32(29)$ \\
\hline Considered treatment related & & & $1(<1)$ \\
\hline \multicolumn{4}{|l|}{ Treatment discontinuation } \\
\hline Due to any $A E$ & & & $9(8)$ \\
\hline Due to any SAE & & & $3(3)$ \\
\hline \multicolumn{4}{|c|}{$\begin{array}{l}\text { AEs considered treatment-related occurring in } \geq 3 \% \text { of } \\
\text { patientst }\end{array}$} \\
\hline Nausea & $42(39)$ & $0(0)$ & 42 (39) \\
\hline Fatigue & $21(19)$ & $2(2)$ & $23(21)$ \\
\hline Vomiting & $18(17)$ & $1(<1)$ & $19(17)$ \\
\hline Peripheral edema & $17(16)$ & $1(<1)$ & $18(17)$ \\
\hline AST increased & $9(8)$ & $3(3)$ & $12(11)$ \\
\hline Blood creatinine increased & $12(11)$ & $0(0)$ & $12(11)$ \\
\hline ALT increased & $6(6)$ & $5(5)$ & $11(10)$ \\
\hline Decreased appetite & $10(9)$ & $1(<1)$ & $11(10)$ \\
\hline Diarrhea & $9(8)$ & $0(0)$ & $9(8)$ \\
\hline Anemia & $6(6)$ & $1(<1)$ & $7(6)$ \\
\hline Constipation & $7(6)$ & $0(0)$ & $7(6)$ \\
\hline Dysgeusia & $7(6)$ & $0(0)$ & $7(6)$ \\
\hline Mucosal inflammation & $6(6)$ & $0(0)$ & $6(6)$ \\
\hline Proteinuria & $5(5)$ & $1(<1)$ & $6(6)$ \\
\hline Stomatitis & $5(5)$ & $0(0)$ & $5(5)$ \\
\hline Hyponatremia & $2(2)$ & $3(3)$ & $5(5)$ \\
\hline Pruritus & $5(5)$ & $0(0)$ & $5(5)$ \\
\hline Edema & $4(4)$ & $0(0)$ & $4(4)$ \\
\hline Weight decreased & $4(4)$ & $0(0)$ & $4(4)$ \\
\hline Hyperkalemia & $3(3)$ & $1(<1)$ & $4(4)$ \\
\hline Hypokalemia & $4(4)$ & $0(0)$ & $4(4)$ \\
\hline Back pain & $4(4)$ & $0(0)$ & $4(4)$ \\
\hline Rash (maculopapular) & $3(3)$ & $1(<1)$ & $4(4)$ \\
\hline Peripheral swelling & $3(3)$ & $0(0)$ & $3(3)$ \\
\hline Fluid retention & $3(3)$ & $0(0)$ & $3(3)$ \\
\hline Hypoalbuminemia & $3(3)$ & $0(0)$ & $3(3)$ \\
\hline Hypomagnesaemia & $3(3)$ & $0(0)$ & $3(3)$ \\
\hline Arthralgia & $3(3)$ & $0(0)$ & $3(3)$ \\
\hline Joint swelling & $3(3)$ & $0(0)$ & $3(3)$ \\
\hline Myalgia & $3(3)$ & $0(0)$ & $3(3)$ \\
\hline Lethargy & $3(3)$ & $0(0)$ & $3(3)$ \\
\hline Rash & $3(3)$ & $0(0)$ & $3(3)$ \\
\hline
\end{tabular}

Abbreviations: AE, adverse event; PRCC, papillary renal cell carcinoma; SAE, serious adverse event.

* Patients with multiple events in the same category are counted only once in that category. Patients with events in more than one category are counted once in each of those categories.

†As assessed by the investigator. Includes AEs with an onset date on or after the date of first dose and up to and including 30 days after the date of last dose of savolitinib. Grade of AEs reported according to Common Terminology Criteria for Adverse Events (version 4.03).

\section{AUTHOR CONTRIBUTIONS}

Conception and design: Toni K. Choueiri, Hendrik-Tobias Arkenau, Melanie M. Frigault, Amir A. Handzel, Humphrey Gardner, Shethah Morgan

Administrative support: Toni K. Choueiri

Provision of study materials or patients: Toni K. Choueiri,

Hendrik-Tobias Arkenau, Elizabeth Plimack, Eric Jonasch, Sumanta

Kumar Pal
Collection and assembly of data: Toni K. Choueiri, Hendrik-Tobias Arkenau, Daniel Y.C. Heng, Melanie M. Frigault, Humphrey Gardner, Shethah Morgan

Data analysis and interpretation: All authors

Manuscript writing: All authors

Final approval of manuscript: All authors

Accountable for all aspects of the work: All authors 


\section{REFERENCES}

1. Choueiri TK, Motzer RJ: Systemic therapy for metastatic renal-cell carcinoma. N Engl J Med 376 354-366, 2017

2. Valenca LB, Hirsch MS, Choueiri TK, et al: Non-clear cell renal cell carcinoma, part 2: Therapy. Clin Adv Hematol Oncol 13:383-391, 2015

3. Tannir NM, Jonasch E, Albiges $L$, et al: Everolimus versus sunitinib prospective evaluation in metastatic non-clear cell renal cell carcinoma (ESPN): A randomized multicenter phase 2 trial. Eur Urol 69: 866-874, 2016

4. Siegel RL, Miller KD, Jemal A: Cancer statistics, 2017. CA Cancer J Clin 67:7-30, 2017

5. Delahunt B, Eble JN: Papillary renal cell carcinoma: A clinicopathologic and immunohistochemical study of 105 tumors. Mod Pathol 10:537-544, 1997

6. Leroy $X$, Zini L, Leteurtre $E$, et al: Morphologic subtyping of papillary renal cell carcinoma: Correlation with prognosis and differential expression of MUC1 between the two subtypes. Mod Pathol 15: 1126-1130, 2002

7. Pignot G, Elie $C$, Conquy $S$, et al: Survival analysis of 130 patients with papillary renal cell carcinoma: Prognostic utility of type 1 and type 2 subclassification. Urology 69:230-235, 2007

8. Gontero P, Ceratti G, Guglielmetti $S$, et al: Prognostic factors in a prospective series of papillary renal cell carcinoma. BJU Int 102:697-702, 2008

9. Sukov WR, Lohse CM, Leibovich $B C$, et al: Clinical and pathological features associated with prognosis in patients with papillary renal cell carcinoma. J Urol 187:54-59, 2012

10. Shuch $B$, Amin A, Armstrong AJ, et al: Understanding pathologic variants of renal cell carcinoma: Distilling therapeutic opportunities from biologic complexity. Eur Urol 67:85-97, 2015

11. Albiges $L$, Guegan J, Le Formal $A$, et al: MET is a potential target across all papillary renal cell carcinomas: Result from a large molecular study of pRCC with $\mathrm{CGH}$ array and matching gene expression array. Clin Cancer Res 20:3411-3421, 2014

12. Linehan WM, Spellman PT, Ricketts CJ, et al: Comprehensive molecular characterization of papillary renal-cell carcinoma. N Engl J Med 374:135-145, 2016

13. Birchmeier C, Birchmeier W, Gherardi E, et al: Met, metastasis, motility and more. Nat Rev Mol Cell Biol 4:915-925, 2003

14. Engelman JA, Zejnullahu K, Mitsudomi T, et al: MET amplification leads to gefitinib resistance in lung cancer by activating ERBB3 signaling. Science 316 : 1039-1043, 2007

15. Bardelli A, Corso S, Bertotti A, et al: Amplification of the MET receptor drives resistance to anti-
EGFR therapies in colorectal cancer. Cancer Discov 3:658-673, 2013

16. Raghav K, Morris $V$, Tang $C$, et al: MET amplification in metastatic colorectal cancer: An acquired response to EGFR inhibition, not a de novo phenomenon. Oncotarget 7:54627-54631, 2016

17. Nakajima $M$, Sawada $H$, Yamada $Y$, et al: The prognostic significance of amplification and overexpression of c-met and c-erb B-2 in human gastric carcinomas. Cancer 85:1894-1902, 1999

18. Kammula US, Kuntz EJ, Francone TD, et al: Molecular co-expression of the c-Met oncogene and hepatocyte growth factor in primary colon cancer predicts tumor stage and clinical outcome. Cancer Lett 248:219-228, 2007

19. Tanimoto S, Fukumori T, El-Moula G, et al: Prognostic significance of serum hepatocyte growth factor in clear cell renal cell carcinoma: Comparison with serum vascular endothelial growth factor. J Med Invest 55:106-111, 2008

20. Ma PC, Tretiakova MS, MacKinnon AC, et al: Expression and mutational analysis of MET in human solid cancers. Genes Chromosomes Cancer 47: 1025-1037, 2008

21. Macher-Goeppinger $S$, Keith $M$, Endris $V$, et al: MET expression and copy number status in clear-cell renal cell carcinoma: Prognostic value and potential predictive marker. Oncotarget 8:1046-1057, 2017

22. Salvi $A$, Marchina $E$, Benetti $A$, et al: Germline and somatic c-met mutations in multifocal/bilateral and sporadic papillary renal carcinomas of selected patients. Int J Oncol 33:271-276, 2008

23. Schmidt L, Duh FM, Chen F, et al: Germline and somatic mutations in the tyrosine kinase domain of the MET proto-oncogene in papillary renal carcinomas. Nat Genet 16:68-73, 1997

24. Schmidt L, Junker K, Nakaigawa N, et al: Novel mutations of the MET proto-oncogene in papillary renal carcinomas. Oncogene 18:2343-2350, 1999

25. Choueiri TK, Plantade A, Elson P, et al: Efficacy of sunitinib and sorafenib in metastatic papillary and chromophobe renal cell carcinoma. J Clin Oncol 26: 127-131, 2008

26. Lee JL, Ahn JH, Lim HY, et al: Multicenter phase II study of sunitinib in patients with non-clear cell renal cell carcinoma. Ann Oncol 23:2108-2114, 2012

27. Molina AM, Feldman DR, Ginsberg MS, et al: Phase II trial of sunitinib in patients with metastatic non-clear cell renal cell carcinoma. Invest New Drugs 30:335-340, 2012

28. Ravaud A, Oudard S, De Fromont $M$, et al: First-line treatment with sunitinib for type 1 and type 2 locally advanced or metastatic papillary renal cell carcinoma: A phase II study (SUPAP) by the French Genitourinary Group (GETUG). Ann Oncol 26: 1123-1128, 2015

29. Tannir NM, Plimack E, Ng C, et al: A phase 2 trial of sunitinib in patients with advanced non-clear cell renal cell carcinoma. Eur Urol 62:1013-1019, 2012

30. Motzer RJ, Bacik J, Mariani T, et al: Treatment outcome and survival associated with metastatic renal cell carcinoma of non-clear-cell histology. J Clin Oncol 20:2376-2381, 2002

31. Motzer RJ, Bacik J, Murphy BA, et al: Interferon-alfa as a comparative treatment for clinical trials of new therapies against advanced renal cell carcinoma. J Clin Oncol 20:289-296, 2002

32. Motzer RJ, Hutson TE, Tomczak $P$, et al: Sunitinib versus interferon alfa in metastatic renal-cell carcinoma. N Engl J Med 356:115-124, 2007

33. Motzer RJ, Hutson TE, Cella D, et al: Pazopanib versus sunitinib in metastatic renal-cell carcinoma. N Engl J Med 369:722-731, 2013

34. Choueiri TK, Vaishampayan U, Rosenberg JE, et al: Phase II and biomarker study of the dual MET/ VEGFR2 inhibitor foretinib in patients with papillary renal cell carcinoma. J Clin Oncol 31:181-186, 2013

35. Oxnard GR, Ramalingam SS, Ahn M-J, et al: Preliminary results of TATTON, a multi-arm phase $\mathrm{lb}$ trial of AZD9291 combined with MEDI4736, AZD6094 or selumetinib in EGFR-mutant lung cancer. J Clin Oncol 33, 2015 (suppl; abstr 2509)

36. Schuller AG, Barry ER, Jones RD, et al: The MET inhibitor AZD6094 (savolitinib, HMPL-504) induces regression in papillary renal cell carcinoma patient-derived xenograft models. Clin Cancer Res 21:2811-2819, 2015

37. Kong Gan H, Lickliter J, Millward M, et al: cMet: Results in papillary renal cell carcinoma of a phase I study of AZD6094/volitinib leading to a phase 2 clinical trial with AZD6094/volitinib in patients with advanced papillary renal cell cancer (PRCC). J Clin Oncol 33, 2015 (suppl; abstr 487)

38. Eisenhauer EA, Therasse $P$, Bogaerts $J$, et al: New response evaluation criteria in solid tumours: Revised RECIST guideline (version 1.1). Eur J Cancer 45:228-247, 2009

39. Frampton GM, Fichtenholtz A, Otto GA, et al: Development and validation of a clinical cancer genomic profiling test based on massively parallel DNA sequencing. Nat Biotechnol 31:1023-1031, 2013

40. Katayama R, Aoyama A, Yamori $T$, et al: Cytotoxic activity of tivantinib (ARQ 197) is not due solely to c-MET inhibition. Cancer Res 73:3087-3096, 2013

41. Schöffski $P$, Garcia JA, Stadler WM, et al: A phase II study of the efficacy and safety of AMG 102 in patients with metastatic renal cell carcinoma. BJU Int 108:679-686, 2011

42. Schoffski P, Wozniak A, Escudier B, et al: Crizotinib achieves objective responses and longlasting disease control in patients (pts) with metastatic papillary renal cell carcinoma type 1 (PRCC1) with somatic MET mutations: EORTC phase II trial 90101 "CREATE". Cancer Res 76, 2016 (abstr CT006)

\section{Affiliations}

Toni K. Choueiri, Dana-Farber Cancer Institute, Boston; Melanie M. Frigault, Edwin A. Clark, Amir A. Handzel, and Humphrey Gardner, AstraZeneca, Waltham, MA; Elizabeth Plimack, Fox Chase Cancer Center, Philadelphia, PA; Eric Jonasch, MD Anderson Cancer Center, Houston, TX; Sumanta Kumar Pal, City of Hope, Duarte, CA; Hendrik-Tobias Arkenau, Sarah Cannon Research Institute, University College London Cancer Institute; Thomas Powles, Barts Cancer Institute, London; Shethah Morgan, AstraZeneca, Cambridge, United Kingdom; Daniel Y.C. Heng, Tom Baker Cancer Center, Calgary, Alberta, Canada; and Laurence Albiges, Institute Gustave Roussy, Paris, France.

\section{Support}

Supported by AstraZeneca, which also funded medical writing support. 


\section{Prior Presentation}

Presented in part at the American Society of Clinical Oncology Genitourinary Cancers Symposium, Orlando, FL, February 16-18, 2017. 
Biomarker-Based Phase II Trial of Savolitinib in Patients With Advanced Papillary Renal Cell Cancer

The following represents disclosure information provided by authors of this manuscript. All relationships are considered compensated. Relationships are self-held unless noted. I = Immediate Family Member, Inst = My Institution. Relationships may not relate to the subject matter of this manuscript. For more information about ASCO's conflict of interest policy, please refer to www.asco.org/rwc or ascopubs.org/jco/site/ifc.

Toni K. Choueiri

Honoraria: National Comprehensive Cancer Network, UpToDate Consulting or Advisory Role: Pfizer, Bayer HealthCare Pharmaceuticals, Novartis, GlaxoSmithKline, Merck, Bristol-Myers Squibb, Genentech, Eisai, Foundation Medicine, Cerulean Pharma, AstraZeneca, Peloton Therapeutics, Exelixis, Prometheus, Alligent

Research Funding: Pfizer (Inst), Novartis (Inst), Merck (Inst), Exelixis (Inst), TRACON Pharmaceuticals (Inst), GlaxoSmithKline (Inst), Bristol-Myers Squibb (Inst), AstraZeneca (Inst), Peloton Therapeutics (Inst), Genentech (Inst), Celldex (Inst), Agensys (Inst)

\section{Elizabeth Plimack}

Consulting or Advisory Role: Novartis, Bristol-Myers Squibb, Acceleron Pharma, Genentech, AstraZeneca, Eli Lilly, Synergene

Research Funding: Bristol-Myers Squibb (Inst), Acceleron Pharma (Inst), AstraZeneca (Inst), Pfizer (Inst), Eli Lilly (Inst), Merck Sharp \& Dohme (Inst), Novartis (Inst), Peloton Therapeutics (Inst)

Patents, Royalties, Other Intellectual Property: US Patent No. 14/ 588,503, filed January 2, 2015 (Inst)

Hendrik-Tobias Arkenau

No relationship to disclose

Eric Jonasch

Consulting or Advisory Role: Pfizer, Novartis, Genentech, Exelixis, Bristol-Myers Squibb, Eisai

Research Funding: Exelixis, Pfizer, Novartis

Daniel Y.C. Heng

Consulting or Advisory Role: Pfizer, Novartis, Bristol-Myers Squibb

Thomas Powles

Honoraria: Novartis

Consulting or Advisory Role: Genentech, Bristol-Myers Squibb, Merck, Novartis, AstraZeneca

Research Funding: AstraZeneca, Genentech,
Melanie M. Frigault

Employment: AstraZeneca

Stock or Other Ownership: AstraZeneca

Patents, Royalties, Other Intellectual Property: AstraZeneca

Edwin A. Clark

Employment: AstraZeneca

Stock or Other Ownership: AstraZeneca

Amir A. Handzel

Employment: AstraZeneca

Leadership: Pangea Diagostics

Stock or Other Ownership: Pangea Diagostics, Kindred Healthcare, Corbus Pharmaceuticals, BG Medicine, Intuitive Surgical

Patents, Royalties, Other Intellectual Property: Pangea Diagostics

\section{Humphrey Gardner}

Employment: AstraZeneca, Karyopharm Therapeutics, Evelo Biosciences Stock Ownership: AstraZeneca, Novartis

\author{
Shethah Morgan \\ Employment: AstraZeneca \\ Leadership: AstraZeneca \\ Stock or Other Ownership: AstraZeneca \\ Honoraria: AstraZeneca \\ Research Funding: AstraZeneca \\ Travel, Accommodations, Expenses: AstraZeneca
}

\section{Laurence Albiges}

Consulting or Advisory Role: Novartis, Pfizer, Sanofi, Amgen,

Bristol-Myers Squibb, Bayer HealthCare Pharmaceuticals

Research Funding: Novartis (Inst), Pfizer (Inst)

\section{Sumanta Kumar Pal}

Honoraria: Novartis, Medivation, Astellas Pharma

Consulting or Advisory Role: Pfizer, Novartis, Aveo, Myriad

Pharmaceuticals, Genentech, Exelixis, Bristol-Myers Squibb, Astellas Pharma

Research Funding: Medivation 


\section{Acknowledgment}

We thank the other investigators who enrolled patients in the study, listed in the Appendix. Medical writing support, funded by AstraZeneca, was provided by Matthew deSchoolmeester, $\mathrm{PhD}$, of Bioscript Medical.

\section{Appendix}

\section{Investigators Enrolling Patients}

The following investigators also enrolled patients in this study: Ulka Vasishampayan (Karmanos Cancer Institute, Detroit, MI), Walter Stadler (University of Chicago, Chicago, IL), Jennifer Knox (Princess Margaret Hospital, Toronto, Ontario, Canada), Balaji Venugopal (Beatson West of Scotland Cancer Centre, Glasgow, United Kingdom), Pablo Maroto (Hospital de Sant Pau, Barcelona, Spain), Howard Burris (Sarah Cannon Research Institute [SCRI] -Tennessee Oncology, Nashville, TN), Lowell Hart (SCRI-Florida Cancer Specialists, West Palm Beach, FL), Kathryn Fife (Addenbrookes and Cambridge University Hospitals, Cambridge, United Kingdom), Georg Bjarnason (Sunnybrook Research Institute, Toronto, Ontario, Canada), Naveen Basappa (University of Alberta Cross Cancer Institute, Edmonton, Alberta, Canada), Robert Hawkins (The Christie Clinic, Manchester, United Kingdom), Sandhya Srinivas (Stanford University, Stanford, CA), Guru Sonpavde (University Alabama at Birmingham, Birmingham, AL), Daniel Vaena (University of Iowa, Iowa City, IA), Ana Molina (Weill Cornell Medicine, New York, NY), and Thomas Hutson (Baylor Sammons Cancer Center, Dallas, TX).

\section{Methods}

Next-generation sequencing (NGS) of archival tumor tissue was analyzed using a targeted 400-gene panel (version T7; Foundation Medicine, Cambridge, MA) as previously described. ${ }^{39}$ Briefly, DNA extraction (a minimum of 50 ng of DNA was required to pass quality-control criteria) and library construction from 40 microns of formalin-fixed paraffin-embedded tumor tissue was used for hybridization capture with probes for each exon of 400 genes, including 23 genes on chromosome 7 and 4,200 single-nucleotide polymorphisms across the genome for proprietary analytics that determined the purity and ploidy of the sample genome, chromosome 7 copy number relative to the genome ploidy (where one additional copy including the MET locus was considered chromosome 7 gain), and MET kinase domain mutations and MET or HGF gene focal amplification.

MET mutations previously identified in papillary renal cell carcinoma (PRCC) were also identified in this trial, and all but one are found in the kinase domain and include (NM_000245) V1092I, H1094L ( $\mathrm{n}=2$ ), L1195F ( $=2$ ), M1131T, and M1250T. One MET mutation was identified outside the kinase domain, V37A, which has not been previously reported (Durinck S, et al: Nat Genet 47:13-21, 2015 $)^{11,12}$ and was not associated with savolitinib response; therefore, only kinase domain MET mutations were considered MET driven for this trial. The copy number range for focal MET locus amplification ( $<20 \mathrm{Mb}$ by targeted NGS) identified in this PRCC trial was three, seven $(n=2)$, eight $(n=2)$, nine, 10 , and 12 , where patient samples harboring \pm seven copies, but not three copies, correlated with savolitinib response. Furthermore, the NGS assay has been analytically validated for six copies as a cutoff for focal amplifications. ${ }^{39}$ Taken together, only focal gains of more than six copies were classified as MET driven in this trial. Focal MET amplifications identified by targeted NGS were confirmed by fluorescent in situ hybridization (FISH). MET FISH has been used as a method for patient selection to identify MET-amplified tumors in other indications, such as non-small-cell lung cancer, gastric cancer, and colorectal cancer (Cappuzzo F, et al: J Clin Oncol 27:1667-1674, 2009; Kawakami H, et al: Oncotarget 4:9-17, 2013), ${ }^{15}$ and has been clinically validated as a tool for other receptor tyrosine kinase-driven diseases, such as human epidermal growth factor receptor 2 in breast cancer (Slamon D, et al: N Engl J Med 365:1273-1283, 2011), and therefore served as a robust orthogonal method to confirm the MET amplification findings in the NGS profiling or PRCC tumor samples from this trial. 
Choueiri et al

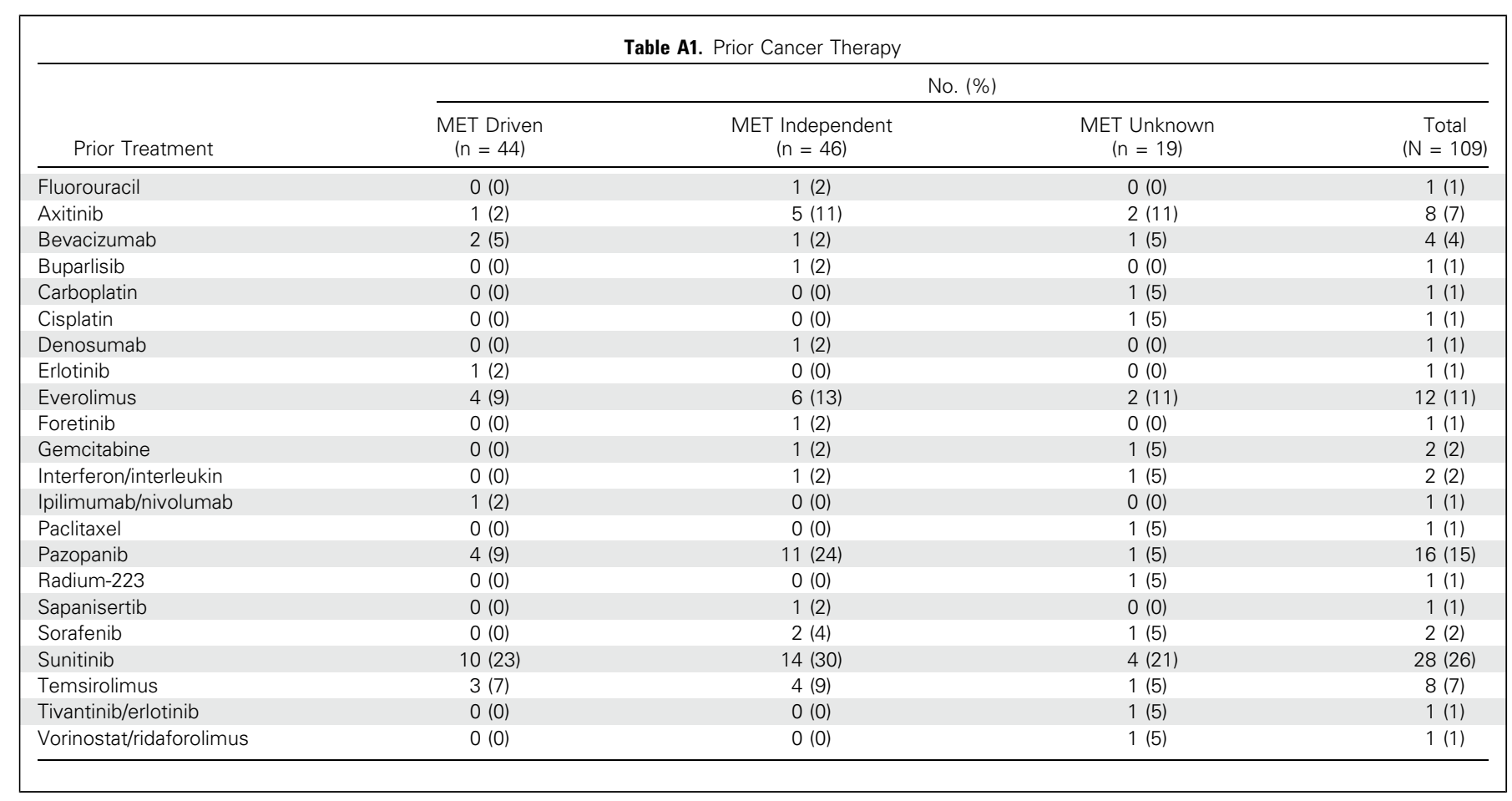

\begin{tabular}{|c|c|c|c|c|c|c|c|c|}
\hline \multirow[b]{2}{*}{ Cycle } & \multicolumn{2}{|c|}{ MET Driven } & \multicolumn{2}{|c|}{ MET Independent } & \multicolumn{2}{|c|}{ MET Unknown } & \multicolumn{2}{|c|}{ Total } \\
\hline & No. & Mean (SD) & No. & Mean (SD) & No. & Mean (SD) & No. & Mean (SD) \\
\hline \multicolumn{9}{|l|}{ FACT-G } \\
\hline 2 & 19 & $5.1(9.8)$ & 18 & $-4.6(8.6)$ & 10 & $-2.7(10.4)$ & 47 & $-0.3(10.3)$ \\
\hline 7 & 6 & $8.7(13.2)$ & 2 & $-6.0(2.8)$ & 1 & 1.0 & 9 & $4.6(12.4)$ \\
\hline 13 & 1 & 21.8 & 1 & -1.0 & 0 & 0 & 2 & $10.4(16.1)$ \\
\hline \multicolumn{9}{|l|}{ FKSI-19 } \\
\hline 2 & 34 & $2.3(8.8)$ & 34 & $-3.1(8.5)$ & 16 & $-3.1(6.8)$ & 84 & $-0.9(8.7)$ \\
\hline 7 & 15 & $3.9(9.0)$ & 4 & $-6.6(6.0)$ & 2 & $1.5(6.4)$ & 21 & $1.7(9.1)$ \\
\hline 13 & 5 & $9.1(7.5)$ & 2 & $-6.0(5.7)$ & 0 & 0 & 7 & $4.8(9.8)$ \\
\hline \multicolumn{9}{|c|}{ EQ-5D-5L } \\
\hline 2 & 15 & $-6.3(12.2)$ & 17 & $1.0(20.6)$ & 9 & $1.0(5.3)$ & 41 & $-1.7(15.5)$ \\
\hline 7 & 5 & $-9.8(11.8)$ & 2 & $-5.5(0.7)$ & 1 & -5.0 & 8 & $-8.1(9.3)$ \\
\hline 13 & 0 & 0 & 1 & $<1$ & 0 & 0 & 1 & $<1$ \\
\hline
\end{tabular}

